\title{
Balloon 090100001: A short and long period pulsating sdB star ${ }^{\star}$
}

\author{
R. Oreiro ${ }^{1,2}$, F. Pérez Hernández ${ }^{1,2}$, A. Ulla ${ }^{3}$, R. Garrido ${ }^{4}$, R. Østensen ${ }^{5}$, and J. MacDonald ${ }^{6}$ \\ 1 Instituto de Astrofísica de Canarias, 38200 La Laguna, Spain \\ e-mail: ror@iac.es \\ 2 Departamento de Astrofísica, Universidad de La Laguna, 38200 La Laguna, Spain \\ e-mail: fph@iac.es \\ 3 Departamento de Física Aplicada, Universidade de Vigo, 36200 Vigo, Spain \\ e-mail: ulla@uvigo.es \\ ${ }^{4}$ Instituto de Astrofísica de Andalucía, 18008 Granada, Spain \\ e-mail: garrido@iaa.es \\ 5 Isaac Newton Group of Telescopes, 37800 Santa Cruz de La Palma, Spain \\ e-mail: roy@ing.iac.es \\ ${ }^{6}$ Department of Physics and Astronomy, University of Delaware, Newark, DE 1976, USA \\ e-mail: jimmacd@udel.edu
}

Received 11 January 2005 / Accepted 1 April 2005

\begin{abstract}
We report the discovery of long period oscillations in the light curve of the bright sdB pulsator Balloon 090100001 . We find at least three peaks in the low frequency amplitude spectrum of the star (at periods 2733, 3665, $4378 \mathrm{~s}$ ) and 10 certain frequencies in the short period range (118-356 s), but probably more than 25 frequencies modulate the light curve of this object. This star is one of only two sdBs, together with HS 0702+6043, known to show simultaneously short and long period pulsations. We also discuss the results of a set of multicolour photometric observations of the target, for which an amplitude-phase diagram is possible for the two dominant peaks. Comparision with theoretical models indicate that the short period peaks correspond to $p$ modes clustered around the fundamental and the first three overtones, while the long period peaks correspond to $g$ modes of high radial order.
\end{abstract}

Key words. stars: early-type - stars: subdwarfs - stars: oscillations - stars: individual: Balloon 090100001

\section{Introduction}

The presence of light variations due to stellar pulsations in hot B subdwarfs (sdBs) has been known since 1997. Kilkenny et al. (1997) discovered the first member of the new class of pulsating objects, originally called EC 14026 stars after the prototype, but now also named the V361 Hya stars. All EC 14026 stars have $T_{\text {eff }}$ and $\log g$ values in the ranges $28400-37500 \mathrm{~K}$ and 5.25-6.11 dex, respectively, but not all sdBs with these physical parameters pulsate (e.g., Billères et al. 2002; but also see Chayer et al. 2004). A typical EC 14026 pulsator shows multiperiodic oscillations with frequencies around $6 \mathrm{mHz}$ and amplitudes of less than $10 \mathrm{mmag}$ (see Kilkenny 2002, for a review). Theoretical studies by Charpinet et al. (1997) demonstrate that these oscillations can be explained as acoustic modes ( $p$ modes) of low degree $\ell$ and low radial

* Based on observations made with the IAC80 telescope operated on the island of Tenerife in the Spanish Observatorio del Teide of the Instituto de Astrofísica de Canarias and in the $90 \mathrm{~cm}$ telescope in the Observatorio de Sierra Nevada. order $n$ excited by an opacity bump due to a local enhancement of iron-group elements.

In 2002, the announcement of another class of pulsating objects among the sdB stars was made by Green et al. (2003). The PG 1716 stars, named after the prototype, have $T_{\text {eff }}$ values in the range $25000-30000 \mathrm{~K}$ and $\log g$ between 5.4 and $5.7 \mathrm{dex}$, they show periods in the range 45 min to $2 \mathrm{~h}$ and amplitudes below 2 mmag. Fontaine et al. (2003) find that unstable gravity modes ( $g$ modes) of high $\ell$ and high $|n|(\ell=3,4 ;|n|>20)$ in models with $T_{\text {eff }}$ lower than $25000 \mathrm{~K}$, driven by the same $\kappa$ mechanism that operates for the EC 14026 stars, provide an explanation for the oscillations.

Schuh et al. (2004) recently reported the presence of long period pulsations in the EC 14026 star HS 0702+6043. This target was then the only sdB known showing simultaneously long and short period oscillations. HS $0702+6043$ is located in the $T_{\text {eff }}-\log g$ plane at the cool end of the instability region of EC 14026 stars, but at the same time at the hot end of the PG 1716 region. If the long period variation is due to independent pulsations, this star would be the first one to simultaneoulsy belong to both classes of $\mathrm{sdB}$ pulsators. However, 
theory does not predict the simultaneous excitation of long period $g$ modes and short period $p$ modes for any sdB model, but instead two different groups separated by a small temperature gap.

Here, we report the detection of similar long period oscillations in the light curve of Balloon 090100001 (hereafter BA09). This object was recently identified as a member of the EC 14026 group in 2004 (Oreiro et al. 2004a; hereafter Paper I) based on the detection of rapid oscillations (frequencies of 2.81 and $3.78 \mathrm{mHz}$ ) in a single five hour photometric run. Here we present the results of seven nights of fast photometry observations that reveal at least three long period oscillations and a more populated and complex amplitude spectrum in the short period range than found in Paper I. This star is also at the cool end of the EC 14026 instability region and at the hot end of the PG 1716 region and, just as HS $0702+6043$, poses a problem to the current theory regarding the excitation mechanism involved. We also present the results of multicolour photometry and discuss the difficulties we find with the interpretation of the obtained results and the potential of BA09 as a target for a multicolour study in order to identify the degree and azimuthal order of its pulsating modes. An extensive multicolour observation has been recently submitted by Baran et al. (2005).

\section{Observations}

After the discovery of short period oscillations in the light curve of BA09 in September, 2003 (see Paper I) we planned a longer observational campaign to more precisely determine the amplitude spectrum of the star, as only two independent frequencies (at 2.8 and $3.7 \mathrm{mHz}$ ) had been detected. Moreover, we organized a multicolour observational campaign to obtain information on the degree $\ell$ of the oscillations. We describe the $B$ filter observations obtained at the Teide Observatory in Sect. 2.1 and the multicolour photometry observations made at the Sierra Nevada Observatory in Sect. 2.2.

\subsection{Fast photometric observations in B filter}

We used the Troms $\varnothing$ CCD Photometer (TCP, see Østensen \& Solheim 2000) attached to the $80 \mathrm{~cm}$ IAC80 telescope (Teide Observatory) to observe the pulsations of BA09. The $B$ filter, and a cycle time of $10 \mathrm{~s}$ were employed for this purpose. The observational campaign ran from August, 12 to 18th, 2004, plus an aditional night obtained on September, 9th. The log of the observations is shown in Table 1.

The data were reduced with the Real Time Photometry (RTP) software (see Østensen 2000): sky subtraction, division by a comparison star and a third order polynomial fitting were applied to each run in order to obtain the light curve of the star. This latter polynomial fit changes the amplitudes of the low frequency peaks discussed later by less than $3 \%$. The resulting curve is shown in Fig. 1. The top panel corresponds to the complete BA09 light curve, while the bottom one shows a detail of a representative night. A synthetic light curve generated from the derived frequencies is overplotted as a continous line. In the top panel a large beating effect is evident, which is due to the presence of two close frequencies, as discussed later.
Table 1. Date and UT of the start of each run together with its corresponding length for the IAC80 data.

\begin{tabular}{lcc}
\hline \hline Date & UT start time & Length \\
\hline August 12, 2004 & $22: 29: 20$ & $6 \mathrm{~h} 57 \mathrm{~m} 40 \mathrm{~s}$ \\
August 13, 2004 & $23: 23: 40$ & $16 \mathrm{~m} 20 \mathrm{~s}$ \\
August 13, 2004 & $23: 44: 40$ & $5 \mathrm{~h} 38 \mathrm{~m} 10 \mathrm{~s}$ \\
August 14, 2004 & $21: 57: 20$ & $7 \mathrm{~h} 34 \mathrm{~m} 30 \mathrm{~s}$ \\
August 15, 2004 & $22: 03: 30$ & $3 \mathrm{~h} 28 \mathrm{~m} 10 \mathrm{~s}$ \\
August 16, 2004 & $21: 56: 00$ & $7 \mathrm{~h} 41 \mathrm{~m} 10 \mathrm{~s}$ \\
August 18, 2004 & $00: 11: 40$ & $5 \mathrm{~h} 23 \mathrm{~m} 40 \mathrm{~s}$ \\
September 9, 2004 & $20: 50: 20$ & $9 \mathrm{~h} 01 \mathrm{~m} 20 \mathrm{~s}$ \\
\hline
\end{tabular}

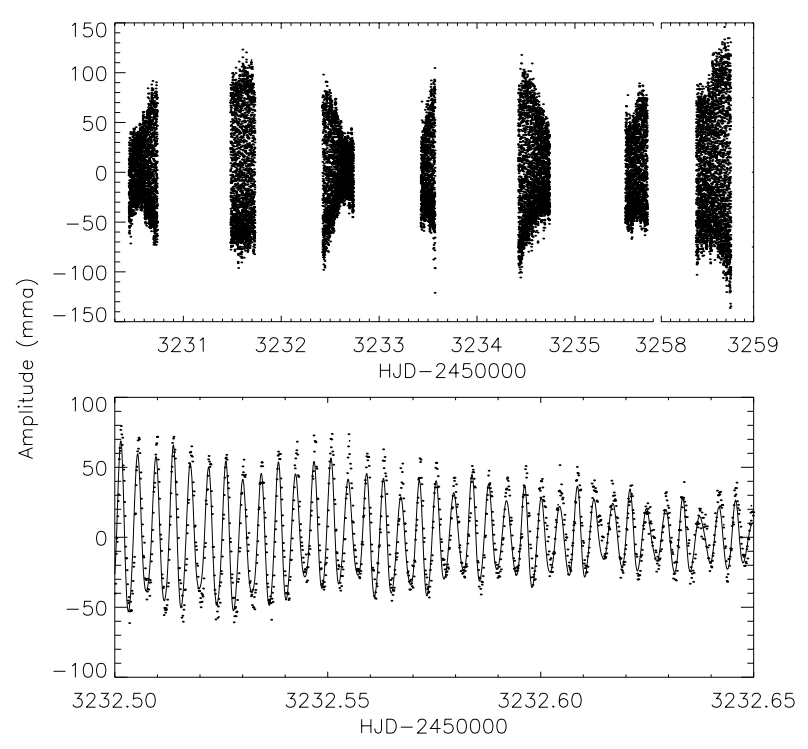

Fig. 1. Top panel: complete normalised light curve of Balloon 090100001. Note that, in order to include the last night a gap in the $x$-axis has been introduced. Bottom panel: a detail from the night of August, 14th (dots) and the synthetic curve obtained from the subsequent analysis (continuous line).

In the bottom panel, a sinusoidal modulation with a period of about ten times the dominant short-period sine is suggested.

The spectral window becomes complicated when the last night of data is included in the analysis, as it is greatly separated from the main bulk of the observations. Despite this complication, this night improves our frequency resolution and allows the detection of a few more frequencies. Figure 2 shows the window structure resulting from the complete data set.

We used an iterative sine wave fitting technique (Ponman 1981) to obtain the amplitude spectrum of the star from the observed light curve. We have also used a multifrequency analysis (Breger et al. 1993) with similar results, as will be commented later. The results of the frequency analysis is summarized in Fig. 3, where the upper most panel corresponds to the amplitude spectrum of BA09, and the following ones display the residuals after the subtraction of 5, 10, 15, 20 and 32 frequencies respectively. We used the prewhitening technique to carry 


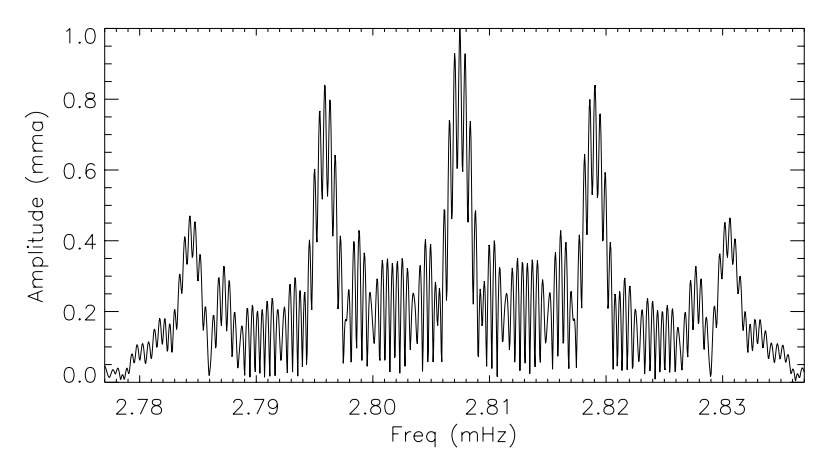

Fig. 2. Spectral window resulting from the complete observations.

out the successive elimination of frequencies in the signal. The spectral region above $9 \mathrm{mHz}$ has not been plotted as no significant peaks exist in this frequency range. In all these panels, the continuous horizontal lines gives 3.7 times the noise level, calculated by averaging the amplitudes over the full frequency range. A suitable extinction correction was applied in order to reduce the noise at very low frequencies, but no significant improvements resulted compared to the amplitude spectrum shown in Fig. 3

The frequency resolution is considerably improved compared to that obtained in Paper I $(63 \mu \mathrm{Hz})$, as we now obtain a full width at half maximum of $2 \mu \mathrm{Hz}$. As a result, some differences arise in the amplitude spectrum of BA09 compared to that obtained in Paper I. The dominant frequency, around $2.8 \mathrm{mHz}$, now has an amplitude of $49.9 \mathrm{mma}$, against the 59.6 mma found in Paper I. The higher modulation could be easily explained if we were observing the star in a constructive beating phase. We find again power around $3.7 \mathrm{mHz}$ and the first harmonic of the dominant peak (around $5.6 \mathrm{mHz}$ ), but now we are able to detect signal at $4.6 \mathrm{mHz}$ and $6.5 \mathrm{mHz}$; furthermore, the first harmonic now appears to be a double peak, and we confirm the presence of some power at low frequencies (around $0.3 \mathrm{mHz}$ ) suggested by the long-period modulation seen in Fig. 1, which was cautiously attributed to atmospheric fluctuations in Paper I. In addition, the second harmonic of the dominant peak $(8.4 \mathrm{mHz})$ can be seen in the present amplitude spectrum.

As the prewhitening process can introduce spurious signal below some amplitude level, and possible errors can occur in the lateral bands even in the first prewhitening step, with consequent error accumulation in the following prewhitening phases, we do not consider all 32 frequencies eliminated in the bottom panel of Fig. 3 as confident, but only those of them listed in Table 2. In this table of confident peaks no more than two or three frequencies from each of the excited frequency ranges has been taken. Having in mind future observations and possible frequency comparisons, we list in Table 3 the "not confident" frequencies. These are all above 3.7 times the noise level. We show in Fig. 4 (and Fig. 5) the complexity of the residuals after two (three) frequencies are removed in the frequency range of the dominant (long period) oscillation. This indicates that we are not able to resolve the presence of very close frequencies.

Data analysis using the simultaneous multiple-frequency technique described in Breger et al. (1993) yields the same confident frequencies of Table 2, whereas slight differences regarding the frequencies listed in Table 3 are found.

\subsection{Fast photometric observations in uvby filters}

In addition to the data gathered at the Teide Observatory, we obtained observing time at the Sierra Nevada Observatory to use the Strömgren photometer attached to the $90 \mathrm{~cm}$ telescope. This instrument allows simultaneous measurements in the $u, b, v, y$ filters. The details of the multicolour observations are given in Table 4.

The complexity, or simplicity in some cases, of the observed amplitude spectra may obstruct a theoretical mode identification in sdBs. Some channels explored so far to get the information on the eigenfunction quantum numbers $(\ell, m)$ needed to carry out the seismological study include: observational-theoretical frequency matching (e.g. Brassard et al. 2001); spectroscopic time series (O'Toole et al. 2000); multicolour photometry (Koen 1998; Koen et al. 2004; Jeffery et al. 2004); or simultaneous time-series spectroscopy and multicolour photometry (Falter et al. 2003). Since BA09 represents a very suitable target for a mode identification due to its brightness and high oscillation amplitude, we chose simultaneous multicolour photometry to undertake a mode identification of the BA09 pulsations by using the method described in Moya et al. (2004), in which non-adiabatic oscillations, including not only the interior but also the atmosphere, are computed. For determination of amplitudes and phases this has been shown to be very important and permits avoiding the use of free parameters. Baran et al. (2005) have also undertaken a multicolour photometry study of the same target obtaining amplitudes and phases for the $U B V R$ filters.

Unfortunately, with our few data sets, the signal to noise ratio only allows detection of two peaks using the same method as with the Teide data. In the top panel of Fig. 6 we show the spectral window of the OSN observations. It was generated from a single sine function with the same frequency and amplitude as the first peak prewhitened in the $u$ amplitude spectrum. The bottom panel corresponds to the amplitude spectra of the $u$ and $y$ bands in the frequency range of interest. Although within the frequency resolution two peaks cannot be distinguished, all the bands show the same asymmetric peak in contrast to the window shape. Also, we find that the amplitude of the first peak is slightly higher in the $u$ band than in the other filters.

In Table 5 we summarize the amplitudes and phases in the different wavebands, obtained by keeping the frequency fixed to that indicated in the first row of the table, which corresponds to the maxima in the $u$ band. The errors shown in Table 5 are the result of a least squares fit to sine functions with the given frequencies. From the table, an increase in amplitude towards bluer wavelengths can be observed for the main frequency peak at $v_{A}$, with $A(u)$ particularly higher than those in the other filters. The amplitudes at frequency $v_{B}$, however, cluster around one value, though here we find the maximum amplitude in the $v$ filter. Concerning the phases, for $v_{A}$ they seem to be similar, while for $v_{B}$ we find a slightly different phase for the $u$ band (see Table 5). 


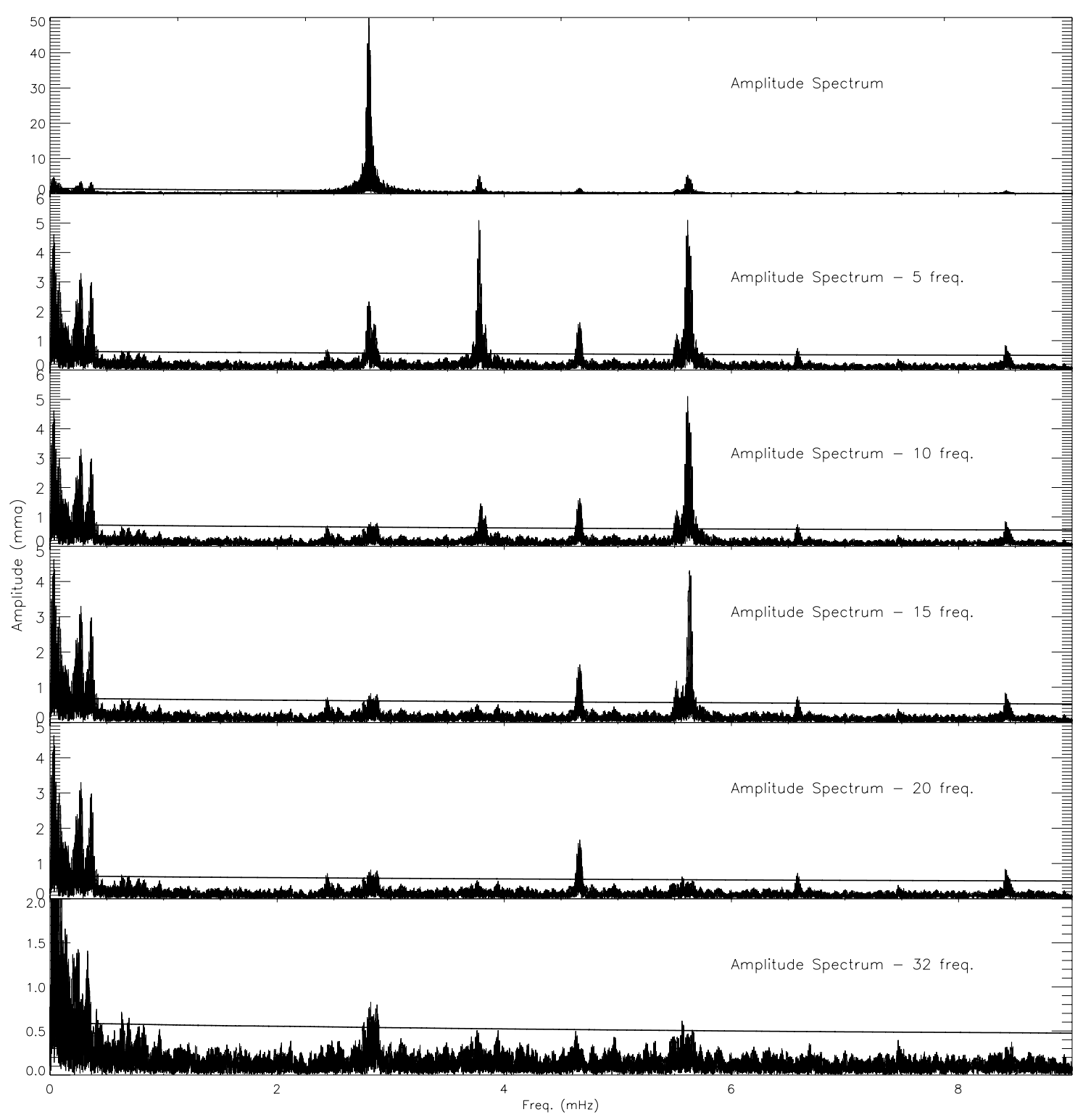

Fig. 3. Top panel: amplitude spectrum obtained from the complete light curve of BA09. From second to bottom panels: residual amplitude spectrum after subtracting 5,10,15, 20 and 32 frequencies respectively. The horizontal line in each panel indicates 3.7 times the noise level.

Considering $u$ and $y$ filters, we have constructed an amplitude-phase diagram where the amplitude ratio $(u-y) / y$ is plotted as a function of the corresponding phase difference for the two peak frequencies listed in Table 5. A substantially different behaviour for the two frequencies is found, suggesting a different $\ell$ value. However, as these two frequencies do not match within errors the results found in the previous subsection, we remind that the frequency resolution does not allow to resolve for example $v_{4}$ and $v_{5}$ in Table 2, and so the amplitudes and phases may change considerably with a better resolution. We are awaiting a longer multicolour observation to continue the present analysis.

\section{Discussion}

Following Oreiro et al. (2004b), the stellar models covering the location of BA09 in the HRD have a fundamental mode $(\ell=0, n=1)$ with a frequency in the range 1.51 to $2.22 \mathrm{mHz}$. Considering those models, the frequencies $v_{4}$ to $v_{13}$ in Table 2 and $v_{b}$ to $v_{q}$ in Table 3 can be explained as $p$ modes with radial orders from $n=1$ up to $n=11$. However, for a given model, the range of $n$ spanned by these periods is much shorter.

In order to investigate this point further we shall consider here some specific models. In particular Fig. 7 shows the location of BA09 in the HRD together with two evolutionary tracks of sdB stars. These tracks were produced by evolving $1.0 M_{\odot}$ model stars from the main sequence through the helium core flash and on to the white dwarf cooling track using the stellar evolution code of one of us (JM) (Jiménez \& MacDonald 1996). Only part of the complete tracks are shown in Fig. 7. In the evolutionary sequences breathing pulses at the end of core helium burning have not been suppressed, even though their physical significance is questionable. Mass loss on the 
Table 2. Confident frequencies derived from the prewhitening process. Phases start at HJD 2453 230.44076. The frequency resolution, computed as indicated in the text, is $2 \mu \mathrm{Hz}$. Combination frequencies identified are indicated in the last column.

\begin{tabular}{rrrrrrl}
\hline \hline & $\begin{array}{r}\text { Freq. } \\
(\mu \mathrm{Hz})\end{array}$ & $\begin{array}{r}\text { Period } \\
(\mathrm{s})\end{array}$ & $\begin{array}{r}\text { Amp. } \\
(\mathrm{mma})\end{array}$ & $\begin{array}{r}\text { Phase } \\
(\mathrm{rad})\end{array}$ & $S / N$ & \\
\hline$v_{1}$ & 228.38 & 4378.7 & 2.25 & 2.925 & 13.2 & \\
$v_{2}$ & 272.80 & 3665.7 & 3.48 & -2.088 & 20.5 & \\
$v_{3}$ & 365.79 & 2733.8 & 2.86 & -2.789 & 16.8 & \\
$v_{4}$ & 2807.46 & 356.2 & 49.88 & 1.961 & 142.5 & \\
$v_{5}$ & 2823.64 & 354.1 & 21.07 & -1.762 & 78.0 & \\
$v_{6}$ & 3776.08 & 264.8 & 5.33 & -0.593 & 31.3 & \\
$v_{7}$ & 3792.22 & 263.7 & 1.50 & 0.247 & 8.8 & \\
$v_{8}$ & 4651.12 & 215.0 & 1.24 & -1.541 & 7.3 & \\
$v_{9}$ & 4664.88 & 214.4 & 1.67 & 2.703 & 10.3 & \\
$v_{10}$ & 5517.99 & 181.2 & 0.95 & 0.734 & 5.6 & \\
$v_{11}$ & 5614.91 & 178.1 & 5.35 & 2.472 & 31.5 & $2 v_{4}$ \\
$v_{12}$ & 5631.52 & 177.6 & 4.57 & -1.789 & 26.8 & \\
$v_{13}$ & 6583.51 & 151.9 & 0.76 & -0.247 & 4.5 & $v_{4}+v_{6}$ \\
$v_{14}$ & 8422.38 & 118.7 & 0.91 & 3.119 & 5.4 & $3 v_{4}$ \\
\hline
\end{tabular}

RGB with Reimer's parameter $\eta=0.680$ and 0.685 result in tracks A and B respectively, whose physical parameters at the beginning of the He-core burning phase are given in Table 6 . From the figure it is seen that the target is well reproduced by track B, which matches the stellar parameters $\log g$ and $T_{\text {eff just }}$ after the end of core helium burning.

To avoid uncertainties in the mean density of the star we consider frequency ratios of $p$ modes as illustrated in Fig. 8. Here we show the frequency ratios refered to the fundamental mode, $v_{\mathrm{f}}$, as function of time for the two evolution sequences indicated in Fig. 7. For clarity, we show only radial modes. As can be seen, these ratios change very little during the evolution of the sdB models.

We now consider the observed frequencies $v_{4}, v_{6}$ and $v_{8}$ which are not only independent peaks but the dominant ones in their corresponding frequency bumps. For the frequency bump around $5.6 \mathrm{mHz}$ the dominant peak is the first harmonic of $v_{4}$ and so we choose the independent frequency $v_{10}$ in this case. Their ratios are compared with the theoretical ones in Fig. 8. As we do not know the age of the star, the time position for the observed frequency ratios (diamond symbols) in Fig. 8 has no significance. The agreement between the observed and theoretical ratios is clear. In fact it is tentative to identify $v_{4}$ as the fundamental radial mode and $v_{6}, v_{8}$ and $v_{10}$ as the first, second and third radial overtones. However, if rather than $v_{4}$ we choose from Table 2 a close frequency as the fundamental radial mode, a rather similar result can be found. Also, non-radial oscillations have similar ratios although in this case the presence of mixed modes masks the corresponding figures. Despite the large number of frequencies detected, which implies that nonradial modes must be present, Fig. 8 suggests that all the short
Table 3. Not confident frequencies derived from the prewhitening process in order to reduce the signal below 3.7 times the noise level. The frequency resolution, computed as indicated in the text, is $2 \mu \mathrm{Hz}$.

\begin{tabular}{cccccc}
\hline \hline & $\begin{array}{c}\text { Freq. } \\
(\mu \mathrm{Hz})\end{array}$ & $\begin{array}{c}\text { Period } \\
(\mathrm{s})\end{array}$ & $\begin{array}{c}\text { Amplitude } \\
(\mathrm{mma})\end{array}$ & $\begin{array}{c}\text { Phase } \\
(\mathrm{rad})\end{array}$ & $S / N$ \\
\hline$v_{a}$ & 331.23 & 3019.0 & 1.43 & -0.507 & 8 \\
$v_{b}$ & 2441.66 & 409.5 & 0.75 & -1.260 & 4.4 \\
$v_{c}$ & 2788.01 & 358.7 & 1.54 & -1.658 & 9.0 \\
$v_{d}$ & 2807.97 & 355.7 & 2.58 & 1.006 & 15.2 \\
$v_{e}$ & 2810.94 & 355.7 & 5.51 & 0.023 & 29.0 \\
$v_{\mathrm{f}}$ & 2825.21 & 353.9 & 7.69 & 2.586 & 33.4 \\
$v_{g}$ & 2826.34 & 353.8 & 3.43 & -0.721 & 18.0 \\
$v_{h}$ & 2853.07 & 350.5 & 1.40 & 2.116 & 8.2 \\
$v_{i}$ & 2857.93 & 349.9 & 0.95 & 1.374 & 5.6 \\
$v_{j}$ & 3795.39 & 263.5 & 1.02 & -0.811 & 6.0 \\
$v_{k}$ & 3798.60 & 263.2 & 0.83 & -2.349 & 4.9 \\
$v_{l}$ & 3834.53 & 260.8 & 0.97 & -1.859 & 5.7 \\
$v_{m}$ & 4660.47 & 214.6 & 0.65 & -0.596 & 3.82 \\
$v_{n}$ & 4669.62 & 214.1 & 0.85 & 1.001 & 5 \\
$v_{o}$ & 5544.41 & 180.4 & 0.79 & 2.907 & 4.6 \\
$v_{p}$ & 5618.40 & 178.0 & 2.19 & 0.545 & 12.9 \\
$v_{q}$ & 5636.57 & 177.4 & 1.06 & 0.069 & 6.2 \\
$v_{r}$ & 8438.94 & 118.5 & 0.75 & -1.192 & 4.4 \\
\hline & & & & &
\end{tabular}

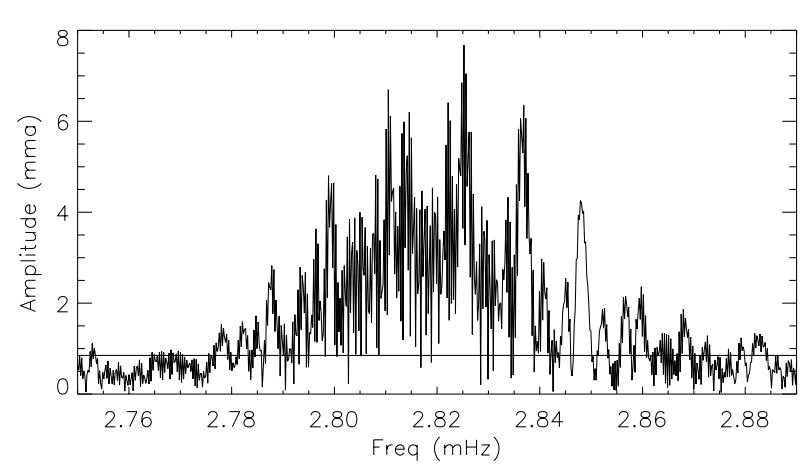

Fig. 4. Residual amplitude spectrum after subtracting 2 frequencies in the frequency range of the main peak.

period peaks are clustered around four consecutive low $n$ values of some degree.

On the other hand, $g$ modes of $|n|>40$ would be necessary to account for the long period oscillations if evolved structural models are considered, while this limit drops to $|n|>20$ for Zero Age Extended Horizontal Branch models. Because these periods are the same order of magnitude as expected, according to Kawaler \& Hostler (2004), for the rotation rates of the cores of sdB stars that evolve as single stars from the RGB, the effects of rotation need to be considered.

Rotational splitting might play a role in the pulsations of the star. As $v_{4}=2807.46, v_{5}=2823.64$ and $v_{h}=2853.07 \mu \mathrm{Hz}$, we find $v_{5}-v_{4}=16 \mu \mathrm{Hz}$ and $v_{h}-v_{5}=2 \times 15 \mu \mathrm{Hz}$. Hence these 


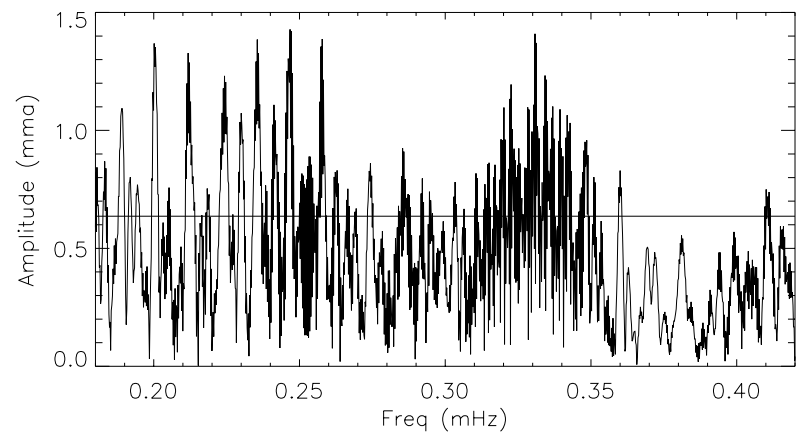

Fig. 5. Residual amplitude spectrum after subtracting 3 frequencies in the frequency range of the low frequency peaks.

Table 4. Details of the OSN data. HJD of the first exposure of each run together with its corresponding length.

\begin{tabular}{cc}
\hline \hline HJD-2 450 000 & Length \\
\hline 3255.33325 & $1 \mathrm{~h} 36 \mathrm{~m} 40 \mathrm{~s}$ \\
3259.35669 & $38 \mathrm{~m} 20 \mathrm{~s}$ \\
3260.33350 & $7 \mathrm{~h} 4 \mathrm{~m} 40 \mathrm{~s}$ \\
\hline
\end{tabular}

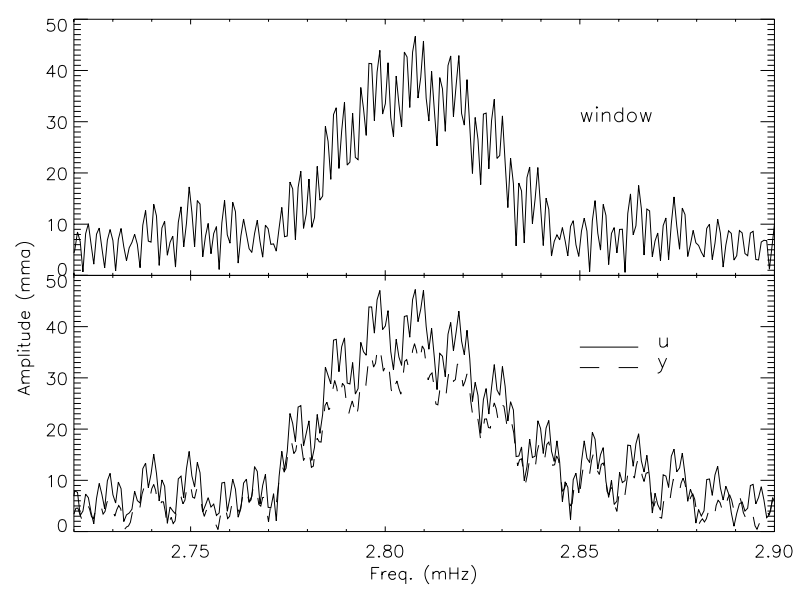

Fig. 6. Top: spectral window corresponding to the OSN observations. Bottom: amplitude spectra in $u$ and $y$ filters in the frequency region of interest.

peaks could correspond to a rotational splitting. Also, as $v_{6}=$ $3776.08, v_{7}=3792.22$ and $v_{l}=3834.53 \mu \mathrm{Hz}$, we have $v_{7}-v_{6}=$ $16 \mu \mathrm{Hz}$ and $v_{l}-v_{7}=3 \times 14 \mu \mathrm{Hz}$, so the $v_{6} ; v_{7} ; v_{l}$ component could also be due to rotational splitting; moreover, $v_{\mathrm{f}}-v_{\mathrm{e}}=$ $14 \mu \mathrm{Hz}, v_{11}-v_{12}=16 \mu \mathrm{Hz}$ and $v_{14}-v_{r}=14 \mu \mathrm{Hz}$. Assuming a radius of $0.2 R_{\odot}$, a frequency splitting of $\Delta v=15 \mu \mathrm{Hz}$ leads to $v_{\text {rot }} \sim 13 \mathrm{~km} \mathrm{~s}^{-1}$, and hence $v \sin i \leq 13 \mathrm{~km} \mathrm{~s}^{-1}$, a result that could easily be confirmed by a specroscopic study.

Establishing the frequencies in Table 3 as secure or not is necessary prior to attempting a detailed seismological study of this object. Moreover, with a longer continuous run, avoiding daily aliases (the possible frequency separation lies relatively close to the 1c/day alias) more frequencies might be detected. A higher resolution and a simpler spectral window are needed to fully take advantage of the seismological potential of BA09.
Table 5. Amplitudes $(A)$, in units of mma, and phases $(\phi)$ in the different colours obtained by fitting the light curve in the corresponding band to a sine function at the given frequencies.

\begin{tabular}{ccc}
\hline \hline Freq. (mHz) & $v_{A}: 2.807$ & $v_{B}: 2.843$ \\
\hline$A(u)$ & $49.36 \pm 0.11$ & $16.73 \pm 0.08$ \\
$A(v)$ & $41.34 \pm 0.11$ & $17.43 \pm 0.09$ \\
$A(b)$ & $38.55 \pm 0.10$ & $16.84 \pm 0.09$ \\
$A(y)$ & $38.03 \pm 0.10$ & $15.76 \pm 0.08$ \\
$\phi(u)[\mathrm{rad}]$ & $-0.710 \pm 0.002$ & $1.619 \pm 0.004$ \\
$\phi(v)[\mathrm{rad}]$ & $-0.706 \pm 0.002$ & $1.740 \pm 0.005$ \\
$\phi(b)[\mathrm{rad}]$ & $-0.698 \pm 0.003$ & $1.730 \pm 0.005$ \\
$\phi(y)[\mathrm{rad}]$ & $-0.703 \pm 0.003$ & $1.725 \pm 0.005$ \\
\hline
\end{tabular}

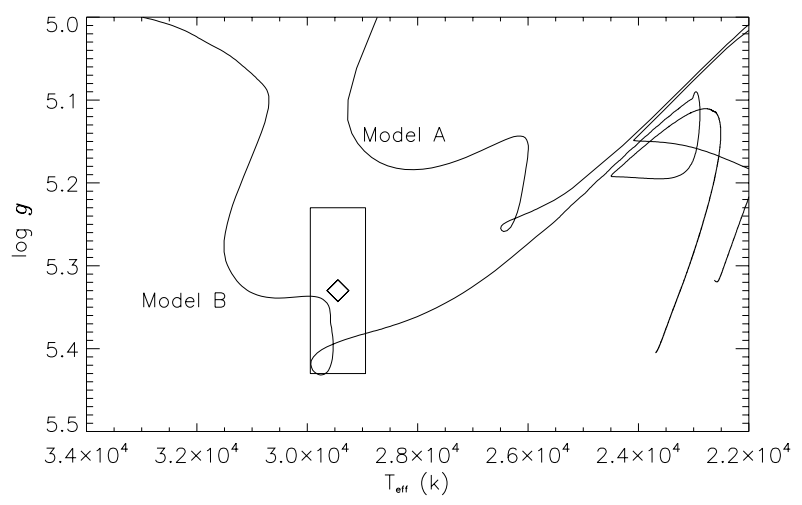

Fig. 7. Theoretical evolutionary tracks for sdB models in the $\log g-$ $T_{\text {eff }}$ diagram. Both tracks correspond to models evolved from the ZAMS as a single stars. In the sdB phase, models $\mathrm{A}$ and $\mathrm{B}$ have a mass of $0.47 M_{\odot}$. The location of BA09 is indicated by a diamond within its error box.

Table 6. Effective temperature $\left(T_{\text {eff }}\right)$, logarithm of surface gravity $(\log g)$, total mass $(M)$ and $\mathrm{H}$-envelope mass $\left(M_{\mathrm{H}}\right)$ of models A and B in Fig. 7 at the beginning of the He-core burning phase.

\begin{tabular}{ccccc}
\hline \hline Model & $T_{\text {eff }}(K)$ & $\log g$ & $M\left(M_{\odot}\right)$ & $M_{\mathrm{H}}\left(M_{\odot}\right)$ \\
\hline A & 22625 & 5.31 & 0.478 & 0.006 \\
B & 23691 & 5.40 & 0.475 & 0.004 \\
\hline
\end{tabular}

\section{Conclusion}

We have obtained $46 \mathrm{~h}$ of fast photometry on the pulsating sdB Balloon 090100001. The results reinforce the seismological interest of this target, as a much more populated spectrum in the frequency domain than that obtained in the first observations has now been found. Moreover, long period oscillations, typical of the PG 1716 subgroup, have been identified. This makes BA09 the second pulsating sdB showing simultaneously both fast and slow ( $\sim 10 \mathrm{~min}, \sim 1 \mathrm{~h}$ ) oscillations (Schuh et al. 2004). However, there are problems in identifying the BA09 pulsating frequencies, due to the presence of very close peaks in its amplitude spectrum. A possible rotational splitting 


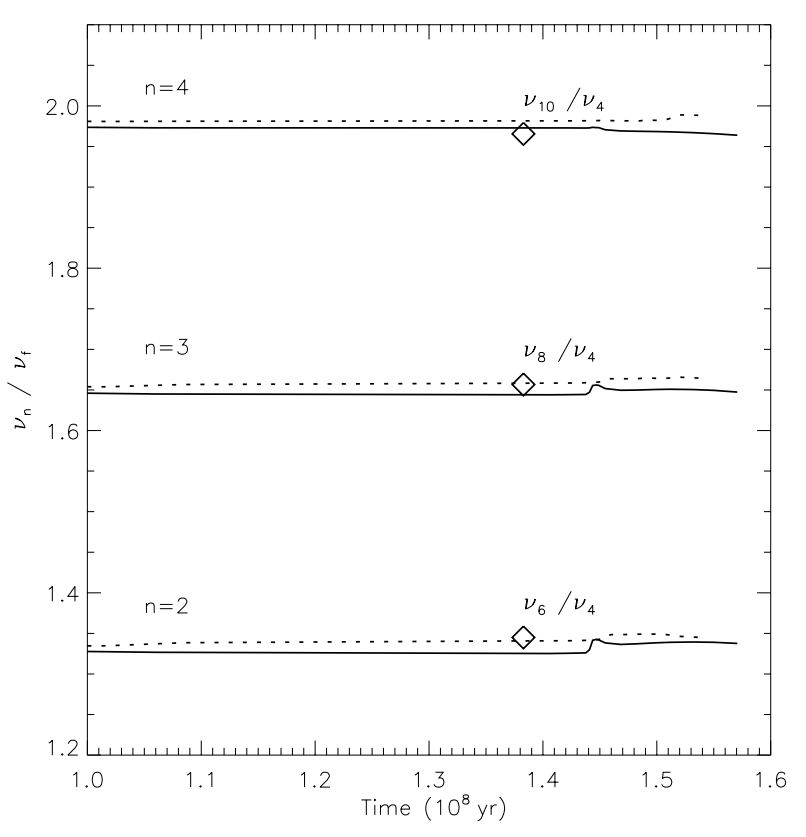

Fig. 8. Frequency ratios for radial modes as function of time. $v_{\mathrm{f}}$ is the frequency of the fundamental radial mode. The continuous lines corresponde to model A and the dashed line to model B. The observed ratios are indicated by diamonds. Here we have considered the higest amplitude frequency $v_{4}$ as if it were the fundamental mode. Time is measured from the ZAHB.

is suggested, but we worry about non-linearities or about the differential rotation hypothesis proposed by Kawaler \& Hostler (2004) in which a rapidly rotating core of an sdB would produce a significantly larger mode splitting than expected from the surface rotational velocity.

We also obtained nine hours of data with a $90 \mathrm{~cm}$ telescope, acquiring simultaneous photometry in the Strömgren $u, b, v, y$ filters. The frequency resolution and signal to noise level achieved allowed us to identify only two peaks at 2807 and $2843 \mu \mathrm{Hz}$. Their amplitudes have a different dependence on wavelength and also the phases have a slightly different dependence on the filter used. This suggests a different $\ell$ value for the modes but, again, a longer multicolour photometry campaign is required to support or reject the validity of such conclusion.

A multi-site campaign would be the best way to resolve the frequency determination and mode identification of the BA09 oscillations. A precise frequency determination is also needed in order to measure possible frequency changes, since, as explained in Paper I, BA09 could be in the final stages of He-core burning, a faster evolutionary state than the stable He-core burning, and would imply a fast evolution of the pulsating properties of the star.
Acknowledgements. This work has been partially funded by grants AYA2001-1571, AYA2003-09499, ESP2001-4529-PE and ESP200403855-C03-03 of the spanish national researh plan. R.O. acknowledges Gobierno de Canarias for its financial support. We also thank CESGA for its computational facilities. J.M. is grateful to Richard Townsend for illuminating conversations regarding pulsating stars. The authors are grateful to the referee, Dr. S. O'Toole, for his useful suggestions.

\section{References}

Baran, A., Pigulski, A., Koziel, D., et al. 2005, MNRAS, submitted Billères, M., Fontaine, G., Brassard, P., \& Liebert, J. 2002, ApJ, 578, 515

Brassard, P., Fontaine, G., Billères, M., et al. 2001, ApJ, 563, 1013

Breger, M., Stich, J., Garrido, R., et al. 1993, A\&A, 271, 482

Charpinet, S., Fontaine, G., Brassard, P., et al. 1997, ApJ, 483, L123

Chayer, P., Fontaine, G., Fontaine, M., et al. 2004, in Extreme Horizontal Branch Stars and Related Objects, ed. P. Maxted, Ap\&SS, 291, 359

Falter, S., Heber, U., Dreizler, S., et al. 2003, A\&A, 401, 289

Fontaine G., Brassard, P., Charpinet, S., et al. 2003, ApJ, 597, 518

Green, E., Fontaine, G., Reed, M., et al. 2003, ApJ, 583, L31

Jeffery, C., Dhillon, V. S., Marsh, T. R., \& Ramachandran, B. 2004, MNRAS, 352, 699

Jiménez, R., \& MacDonald, J. 1996, MNRAS, 283, 271

Kawaler, S., \& Hostler, S. 2004, Ap\&SS, 291, 387

Kilkenny, D., Koen, C., O’Donoghue, D., \& Stobie, R. S. 1997, MNRAS, 285, 640

Kilkenny, D. 2002, in Radial and Nonradial Pulsations as Probes of Stellar Physics, ed. C. Aerts, T. R. Bedding, \& J. Christensen-Dalsgaard (San Francisco: ASP), Proc. IAU Coll., 185,356

Koen, C. 1998, MNRAS, 300, 567

Koen, C., O’Donoghue, D., Kilkenny, D., \& Pollacco, D. L. 2004, NewA, 9, 565

Moya, A., Garrido, R., \& Dupret, M. A. 2004, A\&A, 414, 1081

O'Toole, S., Bedding, R., Kieldsen, H., et al. 2000, ApJ, 537, L53

Oreiro, R., Ulla, A., Pérez Hernández, F., et al. 2004a, A\&A, 418, 243 (Paper I)

Oreiro, R., Ulla, A., Pérez Hernández, F., et al. 2004b, Ap\&SS, 291, 411

Østensen, R. 2000, Time Resolved CCD Photometry, Ph.D. Thesis, University of Troms $\emptyset$, Norway

Østensen, R., \& Solheim, J. E. 2000, BaltA, 9, 411

Ponman, T. 1981, MNRAS, 196, 583

Schuh, S., Huber, J., Green, E., et al. 2004, in 14th Workshop on White Dwarfs, ed. D. Koester, \& S. Moehler, in press 